\title{
Inclusión de nuevas entidades en la toma de decisiones de Aeronáutica Civil en Honduras al año 2015
}

Ana Lucía Ulloa Cadalso

\section{Resumen}

Al hacer un análisis de la Ley de Aeronáutica Civil en Honduras, versión 2004 y que actualmente se encuentra en revisión para su modificación, se establece que el Consejo Aeronáutico Nacional (CAN) debe ser un órgano consultivo que reúne a varias organizaciones estatales y de la sociedad hondureña con la finalidad de identificar, concertar y proponer recomendaciones que apoyen y contribuyan al logro de los objetivos de la ley referida; en la investigación "Consejo Aeronáutico Nacional: Análisis de su situación en el Marco Institucional de Aeronáutica Civil en Honduras, 2014-2015", (Ulloa, 2015) se evidencia que no ha sido activado desde su creación en Ley, incluso en días recientes, con la propuesta de reforma a la Ley de Aeronáutica Civil el CAN desaparece de la misma, llama la atención que en lugar de buscar su implementación el artículo se deroga. Pero esto no quiere decir que las decisiones en relación a diversos temas nacionales tales como seguridad, turismo, medio ambiente, economía, entre otras, que se ven afectados por decisiones que tome la Autoridad Aeronáutica deja de ser relevante. Es importante que los diferentes sectores se coordinen y tomen decisiones consensuadas que permitan un desarrollo sostenido, ordenado y consistente de la aeronáutica civil, aunque no sea a través del CAN. Dado lo anterior, el objetivo del presente artículo es resaltar lo investigado en cuanto a la necesidad latente de incluir a otras entidades en decisiones de Aeronáutica Civil en Honduras al año 2015, para lograr un verdadero desarrollo en materia de Aeronáutica Civil en Honduras.

Palabras clave: Aeronáutica, Consejo Aeronáutico Nacional, aeronáutica civil hondureña, sistema aeroportuario.

\section{Abstract}

Doing an exhaustive analisis of the Civil Aviation Act of Honduras, the 2004 version, we can see that it establishes the National Aviation Council (CAN) as a consultative body that brings together various state organizations and Honduran society in 
order to identify, coordinate and propose recommendations that support and contribute to the achievement of the objectives law referred to in the investigation "National Aviation Council: Analysis of the situation in the Institutional Framework of Civil Aviation in Honduras, 2014-2015" (Ulloa, 2015) is evidence that has not been activated since its inception in law, even in recent days, with the proposed amendment to the Civil Aviation Act cAN disappears from it, draws attention instead to seek implementation repealing article. But this does not mean that decisions concerning various national issues such as security, tourism, environment, economy, among others, that are affected by decisions taken by the Civil Aviation Authority ceases to be relevant. It is important that the different sectors are coordinated and consensual decisions to take sustained, orderly and consistent development of civil aviation, although not through the CAN. Given the above, the objective of this article is to highlight the investigation regarding the latent need to include other entities in decisions of Civil Aviation in Honduras in 2015..

Keywords: Aeronautics, National Aviation Council, Honduran Civil Aeronautics, Airport System.

Ana Lucía Ulloa Cadalso, (ana.ulloa@unah.edu.hn), Departamento de Ciencias Aeronáuticas, Facultad de Ciencias Espaciales Docente, Universidad Nacional Autónoma de Honduras (UNAH). 


\section{INTRODUCCIÓN}

En los últimos años se han observado cambios importantes en el desarrollo de la Aeronáutica Civil en Honduras. Se han habilitado Aeródromos en puntos claves, principalmente a través del mejoramiento y adecuación de Aeródromos militares para uso Civil, sin perjuicio de dicho desarrollo, es necesario asegurarse que este tipo de infraestructura se haya diseñado y opere según los estándares nacionales establecidos para tal efecto por la Agencia Hondureña de Aeronáutica Civil y métodos recomendados internacionales, principalmente, aquellos aeródromos emplazados en aquellos lugares muy próximos a zonas montañosas, resaltando las locaciones de los mismos en ciudades con un alto nivel de atractivo turístico y que permite potenciar el desarrollo económico integral de la zona. Se han llevado a cabo licitaciones para la Concesión del Aeropuerto de Palmerola en Comayagua en aras de convertirlo en un punto clave a nivel nacional, regional e incluso internacional.

Otro dato relevante es la reestructuración de la Autoridad Aeronáutica de Honduras, institución que actualmente se encuentra en un proceso de transición liderado por la Secretaria de Defensa, en aras de capacitar y mejorar el nivel del personal, principalmente técnico, que labora en dicha Institución y con esto permitir el aumento en los niveles de seguridad en el desarrollo de todas las actividades aeronáuticas en el País. El momento actual es oportuno para que las mejorías logradas en la reestructuración antes mencionada, sean un aporte relevante para mejorar la calificación que se le asignará a la Autoridad Aeronáutica Hondureña en la próxima auditoría que lleva a cabo la $\mathrm{OACl}$ bajo el lineamiento $\mathrm{CMA}$ (continuous monitoring approach).

Los proyectos antes mencionados han sido propuestos y desarrollados, principalmente, por iniciativa del Estado de Honduras con la meta de elevar el nivel de la Aeronáutica Civil en el país, aumentar siginificativamente la conectividad nacional y regional, considerando la perspectiva social (llegar lugares para su desarrollo y en caso de ocurrencia desastre natural facilitar rescate y evacuación rápida), promover el turismo y dar una imagen distinta de Honduras a nivel internacional para aumentar el desarrollo económico que se desprende de estas actividades de forma directa 0 indirecta.

Este artículo profundiza aspectos relevantes de la investigación denominada "Consejo Aeronáutico Nacional: Análisis de su situación en el Marco Institucional de Aeronáutica Civil en Honduras, 2014-2015”, (Ulloa, 2015) tomando en consideración el Eje de Investigación \#1: Desarrollo Económico y Social, en el tema prioritario \#1: Infraestructura y Desarrollo Territorial, de la UNAH. Ya el primer artículo presentado 
sobre el CAN enfatiza en la importancia de la participación de los distintos sectores de la aeronáutica para orientar estratégicamente desde una visión amplia de País, la labor de la Autoridad Aeronáutica. Sin embargo, en este mismo trabajo se constata no sólo la inexistencia del Consejo Aeronáutico Nacional, sino la poca 0 nula participación de los sectores relacionados con el transporte aéreo como ser Turismo, Cámara de Hoteleros, Cámara de Comercio, Despacho de Seguridad, Secretaría de Transporte (mencionando algunas de las entidades que formaban parte del CAN según lo descrito por Ley) así como Concesionaria de Aeropuertos, representantes de Línea Aéreas, Escuelas de Aviación e incluso la Universidad Autónoma de Honduras (no incluidas en el CAN según Legislación vigente).

No se considera que estas entidades estén teniendo oportunidad de exponer sus ideas y sus puntos de vista en la toma de decisiones relacionadas con la Aeronáutica Civil y que, de una u otra forma, termina afectándoles. ¿Cuán importante es que estas instituciones, e incluso otras no mencionadas pero, afectadas, participen en la toma de decisiones relacionadas con la Aeronáutica Civil? Es sumamente importante que todos los involucrados puedan tener participación en la toma de decisiones del rubro puesto que es necesario considerar todos los puntos de vista posibles, costos de oportunidad, debilidades y amenazas que enfrenta cada ente independiente, así como el desarrollo de las actividades de cada institución que pueda afectar a otras instituciones o en el mejor de los casos que se puedan tomar decisiones conjuntas utilizando las fortalezas y oportunidades individuales para obtener un beneficio común. Debido a que la Autoridad Aeronáutica de Honduras, posiblemente por estrategia institucional, no está considerando la opción de activar el CAN, elemento que queda evidenciado en la modificación a la Ley de Aeronñautica Civil donde eliminan los artículos relacionados al CAN, es necesario buscar otras formas en que las otras instituciones puedan ser partícipes activos en la toma de decisiones en temas de Aeronáutica Civil.

\section{ANTECEDENTES}

Considerando lo descrito en la Ley de Visión de País (2010-2038) y Plan de Nación (2010-2022) de la República de Honduras donde se expresa: "La infraestructura productiva (energía, transporte y comunicaciones) es un medio importante para propiciar el desarrollo social de los pueblos y el desencadenamiento del potencial productivo de las naciones, Honduras no es la excepción y bajo las circunstancias del país al final del 2009, resulta verdaderamente trascendente iniciar con un 
proceso ordenado, sostenido y consistente de inversiones públicas y privadas, que contribuyan a llevar competitividad, oportunidades de desarrollo económico y social a todas las regiones del país", vemos cuan necesario es el desarrollo de la aeronáutica civil para lograr la visión de país ya que la misma está implícitamente relacionada con los temas de transporte, infraestructura productiva, competitividad y desarrollo económico, al lograr establecer conectividad nacional y regional ofreciendo una perspectiva multimodal de transporte.

En Honduras se han conformado consejos consultivos para distintas finalidades y algunos de ellos continúan vigentes; entre otros ejemplos se pueden mencionar, el que se conformó para dar seguimiento a la Estrategia para la reducción de la Pobreza, documento programático-estratégico diseñado para orientar el desarrollo del País del Año 2000 al 2015; el denominado Consejo Nacional para la Seguridad Interior, CONASIN para el tratamiento de los temas relacionados con el funcionamiento de la Policía Nacional, los Consejos Consultivos Forestales Regionales conformados a instancia del Instituto de Conservación Forestal, por lo que se evidencia que es necesaria para la correcta toma de decisiones, en cualquier ámbito, la incorporación de entidades que muestren distintos puntos de vista, logrando con eso reducir al mínimo los efectos negativos, errores o fracasos de las decisiones tomadas.

En la Ley de Aeronáutica Civil se establece como integrantes del CAN a: Secretario de Estado en el Despacho de Obras Públicas, Transporte y Vivienda (actualmente el Secretario en Infraestructura y Servicios Públicos), quien será el coordinador; el Secretario de Estado en el Despacho de Turismo; el Secretario de Estado en el Despacho de Seguridad; un representante de la Cámara de Turismo; un representante de la Cámara de Hoteleros; un representante de las cámaras de comercio de Honduras; el Director General de Aeronáutica Civil (actualmente Director de la Agencia Hondureña de Aeronáutica Civil) y el Secretario de Estado en el Despacho de Defensa Nacional. En el listado anterior se evidencia el enfoque en la toma de decisiones presupuestarias por parte del Gobierno y podría conseguir la colaboración de una parte del empresariado nacional, más no incluye a la sociedad civil (sociedades de vecinos, patronatos, grupos ambientalistas, grupos de consumidores y demás) ni la Academia (cualquiera de las 6 universidades públicas 0 de las 14 privadas).

Se ha considerado en la investigación en mención que es necesaria la participación activa de un órgano colegiado que integre a diferentes sectores de la institucionalidad 
y sociedad hondureña para apoyar a la Agencia Hondureña de Aeronáutica Civil en la toma de decisiones dentro de su competencia, su fortalecimiento institucional y en gran medida para la gestión de una verdadera política de Estado en materia de aeronáutica civil para el desarrollo nacional y ese órgano colegiado se consideraba debía ser el Consejo Aeronáutico Nacional (CAN).

Como es de conocimiento de muchos la Dirección General de Aeronáutica Civil, ha sido sustituida en el año 2014 por la Agencia Hondureña de Aeronáutica Civil (AHAC) mediante Decreto Ejecutivo PCM-047-2014, quien aporta la visión técnica propia de la aeronáutica civil pero no la económica, social y ambiental. Temas relevantes como, por ejemplo, medidas para la protección y conservación del medio ambiente en la construcción y habilitación de nuevos aeródromos en las diversas regiones del país que requieran de una gestión como Estado y la participación de la sociedad civil deberían ser tratados desde una organización u órgano que aglutine los representantes de los diferentes sectores nacionales desde el estatal al económico y social. Actualmente la mayoría de las decisiones en el ámbito de la Aeronáutica Civil se están tomando desde la institucionalidad del Estado, sin la participación de los diferentes sectores de la sociedad vinculados con la aeronáutica, lo que puede estar afectando el desarrollo del sector.

Es importante mencionar que el tema del CAN en el último año ya no es desconocido por la Autoridad Aeronáutica, que aunque estaba en su Ley era desconocido por sus propios empleados, elemento evidenciado en la investigación; lo que llama mucho la atención es que en la reformas a la Ley de Aeronáutica Civil que se están llevando a cabo (disponibles en http://www.ahac.gob.hn/asesorialegal.html ) lejos de buscar implementar el CAN sugieren la derogación de los artículos 28 y 29 del Capítulo III que son los que hablan específicamente del CAN.

Se desconoce a profundidad las razones por las cuales el CAN se estaría excluyendo en la reforma a la Ley de Aeronáutica Civil, pero se considera puede deberse a estrategias que pretendan readecuar presupuesto y personal a áreas como equipamiento y capacitación especializada del personal, tomando de base para esta presunción las acciones de nuevas contrataciones de personal con un perfil académico más alto y las capacitaciones nacionales e internacionales que, se conoce, periódicamente está llevando a cabo la Autoridad Aeronáutica en Honduras. 


\section{METODOLOGÍA CIENTÍFICA}

La investigación tuvo un enfoque cualitativo pues se pretendió describir la situación del Consejo Aeronáutico Nacional a 2014-2015 en Honduras y por tanto se utilizó "la recolección de datos sin medición numérica para descubrir o afinar las preguntas de investigación en el proceso de interpretación" (Sampieri, 2008). El alcance de la investigación fue descriptivo, buscando especificar propiedades, características y rasgos importantes de cualquier fenómeno que se analiza (Sampieri Hernandez, Fernandez Collado, \& Baptista Lucio, 2010), en este caso particular, la situación del Consejo Aeronáutico Nacional al año 2014-2015, incluyendo sus integrantes y marco normativo. La investigación se llevó a cabo en las ciudades de Tegucigalpa y Comayagüela, limitado a estas ciudades por encontrarse ahí las oficinas administrativas de las entidades relacionadas por Ley con el CAN, sin embargo, el alcance de la conclusiones se proyectaron a nivel nacional.

Una de las técnicas de recolección de datos utilizada fue la observación cualitativa pues mediante la misma se llevó a cabo una reflexión permanente. Se prestó atención a los detalles, sucesos, eventos e interacciones, sobre todo en las discusiones dadas en el marco del Diplomado en Gestión de Sistemas Aeroportuarios en sus tres ediciones, ya que los alumnos coincidían con la importancia de la inclusión de varias entidades en la toma de decisiones en relación a Aeronáutica Civil, identificándose los efectos que una decisión tomada por una entidad puede tener en otras entidades por el simple hecho de desconocer sus procesos internos o metas a corto y largo plazo, por lo que, vuelve a considerarse necesario dar a conocer los distintos puntos de vista de todos los afectados o posibles involucrados para tomar decisiones más acertadas y que impliquen menos costos y mal uso de los recursos.

Es necesario indicar que uno de los instrumentos de recolección de datos mas relevante fue a través del uso de entrevistas abiertas, fundamentadas en una guía general de contenido que incluía principalmente preguntas generales y de opinión y aplicadas a personal específico que formaba parte de Instituciones claves en relación a la Aeronáutica Civil como ser la Concesionaria de los cuatro aeropuertos internacionales de Honduras, la Dirección General de Aeronáutica Civil (actual Agencia Hondureña de Aeronáutica Civil) y representantes del sector turismo. Otras fuentes de recolección de datos utilizadas fueron: Ley de Aeronáutica Civil de Honduras, Leyes y Códigos en materia de aeronáutica civil de países como Chile, Ecuador y República Dominicana disponible en internet, esto pues se consideró 
imprescindible conocer las experiencias que otros países han tenido en este tema con el fin de replicar sus experiencias de éxito. Importante mencionar que durante la investigación base de este artículo, todos los hallazgos fueron discutidos con alumnos con perfiles varios como ser: pilotos, técnicos en mantenimiento de aeronaves, administradores, ingenieros, abogados, empleados de aerolíneas, doctores en medicina relacionada con aeronáutica civil, incluso elementos militares, pudiendo obtenerse un panorama integral de las entidades que tendrían que ser consideradas en la toma de decisiones aeronáuticas.

\section{RESULTADOS}

Dentro de los objetivos específicos de la investigación sobre el CAN se resaltan los siguientes por considerarse oportunos para contestar la pregunta de nuestro artículo: ¿Se debería incluir a otras entidades en decisiones de Aeronáutica Civil en Honduras? Esto nos permite analizar las alternativas, considerando las pocas 0 nulas posibilidades de activar el CAN, para facilitar la participación de los distintos sectores involucrados en la toma de decisiones en tema de Aeronáutica Civil. 
Tabla 1: Resumen de Objetivos relacionados con el tema de análisis y hallazgos de la investigación

\begin{tabular}{|l|l|}
\hline \multicolumn{1}{|c|}{ Objetivo } & \multicolumn{1}{|c|}{ Hallazgos a través de las entrevistas aplicadas } \\
\hline $\begin{array}{l}\text { Determinar si las } \\
\text { entidades, incluidas en el } \\
\text { CAN por medio de la Ley } \\
\text { de Aeronáutica Civil, } \\
\text { están interesadas en } \\
\text { seguir formando parte } \\
\text { del CAN. }\end{array}$ & $\begin{array}{l}\text { La mayoría de los entrevistados indican que, como } \\
\text { representantes de sus instituciones, si estarian interesados } \\
\text { en formar parte activa del CAN. }\end{array}$ \\
\hline $\begin{array}{l}\text { Definir qué otras } \\
\text { entidades podrian formar } \\
\text { parte del CAN para } \\
\text { mejorar su funcionalidad. }\end{array}$ & $\begin{array}{l}\text { En relación a la consulta sobre qué entidades deberían } \\
\text { formar parte del CAN, los entrevistados mencionaron las }\end{array}$ \\
$\begin{array}{l}\text { Industria y Comercio,Empresa privada, Escuelas de } \\
\text { aviación, Ministerio Público. }\end{array}$ \\
\hline $\begin{array}{l}\text { Definir qué temas } \\
\text { debería analizar de } \\
\text { manera inmediata el } \\
\text { CAN }\end{array}$ & $\begin{array}{l}\text { En relación a los temas que se consideran claves para } \\
\text { incluir en la agenda a tratar por el CAN, los entrevistados } \\
\text { mencionan los siguientes: La Categorización del país, } \\
\text { Capacitación de personal técnico aeronáutico, Manejo de } \\
\text { presupuestos y Programas de capacitaciones, Mejoras al } \\
\text { sistema aeroportuario, Planificación de futuras propuestas } \\
\text { de aviación civil, Designación de presupuesto para } \\
\text { actividades específicas de aviación civil, Agenda sobre } \\
\text { supervisiones realizadas, Revisión de metas y objetivos } \\
\text { estratégicos y operacionales, Estudios aeronáuticos para el } \\
\text { desarrollo de la aviación civil, Generación de empleos en el } \\
\text { ambiente aeronáutico, Atención al cliente, Mejora de } \\
\text { instalaciones y equipo, Mejor supervisión de procedimientos } \\
\text { aeronáuticos (plataforma, pistas, instalaciones), Supervisión } \\
\text { a líneas aéreas y a empresas concesionarias con más rigor, } \\
\text { Situación del personal y autonomía de la DGAC. }\end{array}$ \\
\hline
\end{tabular}

Considerando la tabla anterior se evidencia que hay interés en las entidades, descritas en Ley, en formar parte del CAN, están interesados en opinar sobre decisiones que terminan afectándoles directa o indirectamente. 
De igual forma sugieren la inclusión de la Concesionaria, Aerolíneas, Secretaría de Industria y Comercio,Empresa privada, Escuelas de aviación,Ministerio Público, entidades que con un manejo integral e inclusivo permitirían, cubrir el panorama completo del impacto de las decisiones en aeronáutica civil. ¿Por qué considerar a estas entidades adicionales sugeridas para formar parte de un equipo de trabajo, llámese anteriormente CAN? Porque de todos es conocido que cualquier decisión que tome la Autoridad Aeronáutica tiene una implicación para estos; tomemos de ejemplo, AHAC declara un aumento realmente significativo y fuera de período en la tarifa por estacionamiento para aeronaves: esto afectará directamente a la Concesionaria pues es la responsable de hacer el cálculo y cobro directamente a sus clientes recibiendo las quejas y reclamos del mismo por realizarse cobros adicionales fuera del tiempo establecido por Ley, En este mismo caso este cobro dictado por la Autoridad y que debe ser cumplido por la Concesionaria es cobrado a las aeronaves de las aerolíneas, hay molestia por este aumento pero debido a que es una obligación a cumplir las aerolíneas pagan el aumento y para no presentar pérdidas ante sus inversionistas incluyen ese aumento de costos de operación en el precio de sus boletos, hasta este punto se ha afectado directamente al pasajero que tendrá que pagar un precio mayor para realizar sus viajes. ¿Y que ocurre si el precio del boleto es tan alto? Baja la demanda, las aerolínea presentaran pérdida, el turismo nacional puede verse directamente afectado, si baja el turismo baja la rentabilidad de los hoteles y restaurantes y continua la cadena. Lo anterior muestra como una medida que pudiese ser tomada de forma aislada por la Autoridad Aeronáutica y que quizas tenga muy bien justificada la razón de ese aumento, puede afectar a tantos involucrados.

Al no conocerse el punto de vista de todos los demás no podrá considerarse el verdadero impacto económico que causaría a la aeronáutica civil de país. Esto nos ayuda a afirmar que Sí se debería incluir a otras entidades en decisiones trascendentes para la Aeronáutica Civil en Honduras, aunque no sea a través del CAN.

En relación al objetivo Describir cómo funcionan órganos u organizaciones similares al CAN en otros países con mayor desarrollo aeronáutico civil como Chile, República Dominicana y Ecuador, en estos ya se incorporan los distintos sectores de la aeronáutica civil mostrando esquemas de participación amplios que asumen responsabilidades de gran importancia para la aeronáutica civil de los propios países lo que queda evidenciado en el cuadro siguiente: 
Tabla 2a: Resumen de Aspectos relevantes de Órganos similares al CAN que involucran a otras entidades

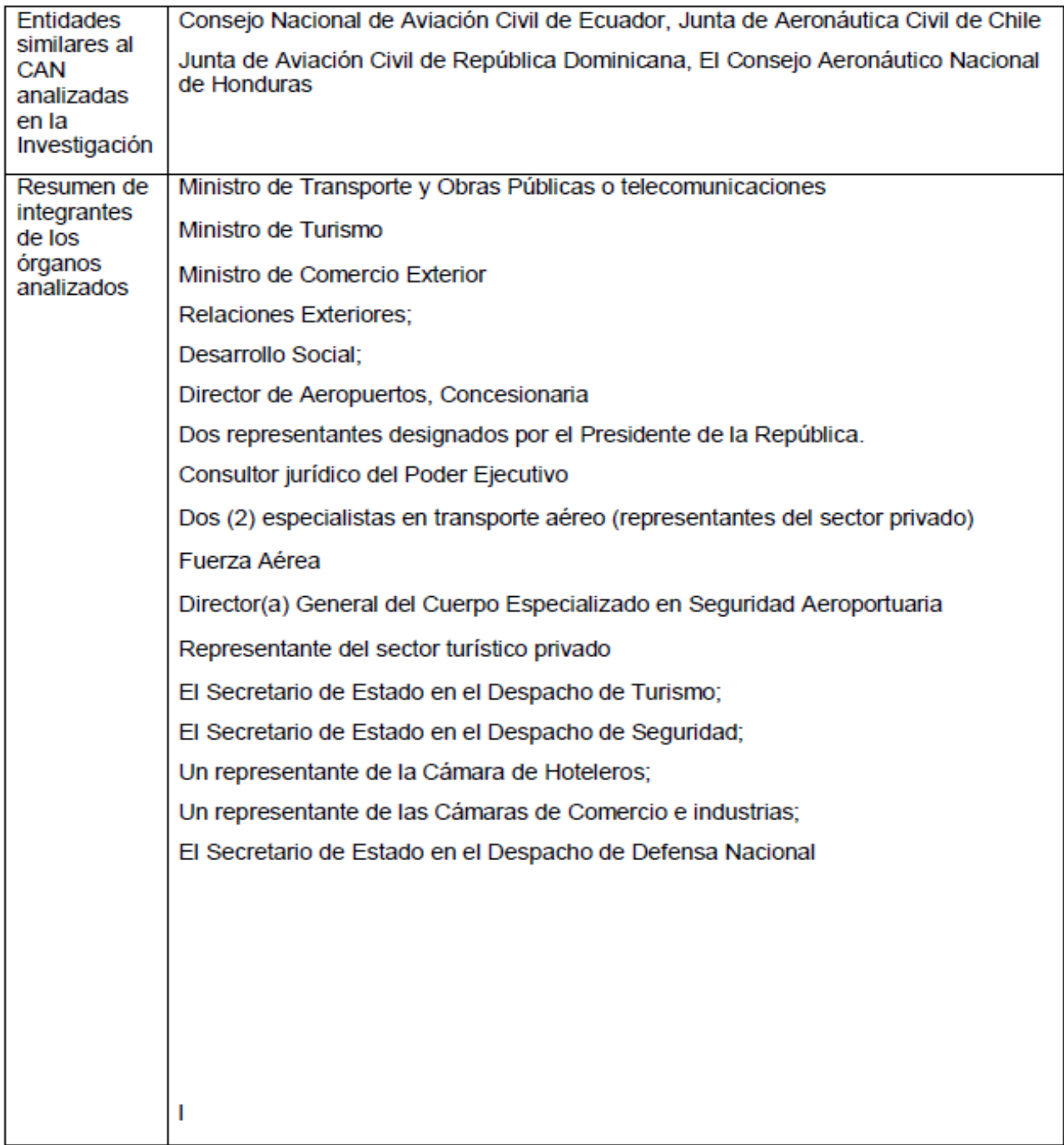

Fuente: Cuadro comparativo de edición propia basado en información disponible en Internet. 
Tabla 2b: Resumen de Aspectos relevantes de Órganos similares al CAN que involucran a otras entidades.

\begin{tabular}{|c|c|}
\hline $\begin{array}{l}\text { Resumen de } \\
\text { Funciones de } \\
\text { los Órganos } \\
\text { analizados }\end{array}$ & $\begin{array}{l}\text { Fomento y desarrollo de la aviación civil comercial } \\
\text { Promoción de un servicio adecuado de las empresas de transporte aéreo } \\
\text { Establecimiento de una red de transporte aéreo como medio de desarrollo } \\
\text { económico } \\
\text { Creación y mantenimiento de los vínculos internacionales mediante la suscripción } \\
\text { de acuerdos y convenios. } \\
\text { Ejercer la dirección superior de la aviación civil } \\
\text { Administrar las políticas de la aviación civil en el país. } \\
\text { Representar al gobierno ante los organismos internacionales en materia } \\
\text { aeronáutica. } \\
\text { Calificar la reciprocidad de derechos que otorguen a las empresas aéreas } \\
\text { nacionales los paises extranjeros. } \\
\text { Promover la facilitación del transporte aéreo internacional. } \\
\text { Proporcionar estadísticas } \\
\text { Registrar las tarifas } \\
\text { Establecer y controlar los seguros de aeronaves comerciales. } \\
\text { Definir las políticas y estrategias para el desarrollo del transporte aéreo en el país. } \\
\text { Proponer al Poder Ejecutivo la adopción de reglamentos relacionados con los } \\
\text { aspectos económicos del transporte aéreo. } \\
\text { Recomendar al Poder Ejecutivo la fijación de tasas y derechos aeronáuticos. } \\
\text { Identificar, concertar y proponer para consideración del Poder Ejecutivo las } \\
\text { recomendaciones que coadyuven al logro de los objetivos de esta Ley, sin que ello } \\
\text { implique quitar atribuciones de vigilancia, reglamentación y regulación con que } \\
\text { cuenta la Dirección General de Aeronáutica Civil; } \\
\text { Conocer los informes anuales y de operación de la Dirección General de } \\
\text { Aeronáutica Civil, emitir opinión sobre los mismos sin perjuicio de las disposiciones } \\
\text { que prescriba la Organización de Aviación Civil Internacional (OACl) sobre emisión } \\
\text { y divulgación de informes sobre actividades de aviación civil de los estados } \\
\text { miembros; } \\
\text { Realizar acciones de control social con respecto a la gestión pública de la } \\
\text { aeronáutica civil promoviendo las acciones respectivas en el marco de la Ley; } \\
\text { Establecer la reglamentación interna para su funcionamiento; y, } \\
\text { Desarrollar actividades de información y divulgación sobre los asuntos de su } \\
\text { competencia y realizar otras acciones de concertación, propuesta y control social } \\
\text { en el marco de la aeronáutica civil. }\end{array}$ \\
\hline
\end{tabular}

Fuente: Cuadro comparativo de edición propia basado en información disponible en Internet. 
Tabla 2c: Resumen de Aspectos relevantes de Órganos similares al CAN que involucran a otras entidades.

\begin{tabular}{|l|l|}
\hline $\begin{array}{l}\text { Logros } \\
\text { alcanzados } \\
\text { por algunos } \\
\text { de estos } \\
\text { órganos } \\
\text { interinstitu- } \\
\text { cionales }\end{array}$ & $\begin{array}{l}\text { Ecuador: re-categorización (2006 categoría 1 luego de haber permanecido en la } \\
\text { categoría 2 desde 1994); } 2007 \text { inició el Plan Nacional de Modernización de } \\
\text { aeropuertos, sistemas de navegación y telecomunicaciones. Construcción del nuevo } \\
\text { aeropuerto de Quito de los más importantes de su región. } \\
\text { liberalizar los cielos, lo que ha generado un marco regulador estable y el cual ha } \\
\text { permitido que los operadores aéreos puedan establecer planes de negocio en } \\
\text { condiciones confiables. }\end{array}$ \\
\hline
\end{tabular}

Fuente: Cuadro comparativo de edición propia basado en información disponible en Internet.

Aunque el cuadro anterior muestra el funcionamiento de Órganos similares al CAN en Honduras, lo importante a resaltar es como la inclusión de distintas entidades, no específicamente aeronáuticas pero que de alguna forma se ven afectadas, con funciones diversas ha permitido el logro de metas significativas en materia de desarrollo de la aeronáutica Civil y es un punto interesante para ser replicado en nuestro país.

Las entrevistas realizadas al Director y Sub Director de la Agencia Hondureña de Aeronáutica Civil mostraron un alto interés en la activación del CAN, indicando que se estaban llevando a cabo cambios en la estructura de la Agencia por la Comisión de Transición de la DGAC a AHAC descrito en Ley, donde hay una mayor participación por parte de la Secretaría de Estado en los Despachos de Seguridad y Defensa pero que estaba dentro de los planes de Aeronáutica Civil la implementación e incluso modificación del CAN según necesidades actuales, posiblemente durante la transición el enfoque sobre el CAN fue modificado y es por eso que aparecen derogados los artículos en las reformas propuestas. Entre los beneficios esperados de una mayor participación de distintos sectores se mencionan los siguientes: la Concesionaria podría estar al tanto de todo lo que pasa en el rubro aeronáutico, se crearían nuevos contactos y conexiones claves, se crearía mayor afinidad con los otros entes involucrados, formación de mejores profesionales para trabajar en la aeronáutica civil, incrementar estándares de seguridad operacional, conformación de SSP y SMS como lo exige la regulación de la aviación civil internacional, parte de las inversiones que se harían serían devueltas a la Concesionaria, se generarían más empleos porque se desarrollarían nuevas áreas para mejorar el servicio en mención. 
Algunos de los temas considerados para analizarse en el CAN y que en este caso pueden tomarse en cuenta en reuniones con otras entidades antes de tomar una decisión aeronáutica están: Categorización del país, capacitación de personal técnico aeronáutico, manejo de presupuestos yiProgramas de capacitaciones, mejoras al sistema aeroportuario, planificación de futuras propuestas de aviación civil, designación de presupuesto para actividades específicas de aviación civil , agenda sobre supervisiones realizadas, revisión de metas y objetivos estratégicos y operacionales, estudios aeronáuticos para el desarrollo de la aviación civil, generación de empleos en el ambiente aeronáutico, atención al cliente, mejora de instalaciones y equipo, mejor supervisión de procedimientos aeronáuticos (plataforma, pistas, instalaciones), supervisión a líneas aéreas y a empresas concesionarias con más rigor, situación del personal y autonomía de la DGAC.

\section{DISCUSIÓN}

La investigacion realizada tenía como enfoque principal hacer un diagnóstico de la situación en que se encontraba el Consejo Aeronáutico Nacional de Honduras y la posibilidad de ser implementado; a medida que se ha considerado, evaluado y analizado el desarrollo de este tema de la mano con el proceso de transición de la Dirección General de Aeronáutica Civil a la Agencia Hondureña de Aeronáutica Civil queda evidenciado que el proceso de implementación no está siendo considerado dentro de las decisiones de la Autoridad Aeronáutica; lo anterior no demerita que continua siendo relevante el tema de inclusión de otras entidades como asesores o simplemente como proporcionadores de opinión de posibles impactos positivos 0 negativos, en cada rubro distinto al aeronáutico que se ve directa o indirectamente afectado por decisiones aeronáuticas.

En base a los argumentos expuestos se continua considerando que es necesario que se incluya a otras entidades, no sólo a instituciones gubernamentales sino que también representantes de la sociedad civil; algunas de las que se sugiere sean consideradas son las cámaras de comercio e industria y cámara de turismo, Universidades, Fuerza Aérea, Concesionaria, Aerolíneas, Secretaría de Industria y Comercio, Empresa privada, Escuelas de aviación, Ministerio Público.

La inclusión de otras entidades debe seguir siendo responsabilidad del Estado, principalmente a través de la Autoridad Aeronáutica con el objetivo de que las decisiones tomadas desde la Dirección estén bien asesoradas. Posiblemente exista resistencia por parte de la misma Autoridad a la inclusión de otras entidades en la toma de decisiones por no querer discutir ciertos temas con organizaciones externas y por tanto no desean la intromisión en sus asuntos, pues podrían 
considerar les producirían complicaciones legales, administrativas, etc, esto parece estar evidenciado en la intención de derogar los artículos de la Ley de Aeronñautica civil que hablan del CAN, pero es posible también que se esté considerando a través de otra estrategia administrativa la inclusión de otras entidades y que sean desconocidas por el autor.

\section{CONCLUSIONES}

El Consejo Aeronáutico Nacional al año 2015 existía solamente según la Ley de Aeronáutica Civil, no se contaba con evidencias de su funcionamiento; y en las reformas a la Ley de Aeronáutica Civil que están siendo propuestas actualmente por la Autoridad Aeronáutica se eliminan los artículos que hablan sobre el CAN por lo que se deduce no hay interés alguno en su implementación o en su defecto la estrategia de la nueva Agencia implica inversión en recursos y personal para otras actividades.

No se tiene información que evidencie la incorporación formal de entidades, distintas a la Autoridad Aeronáutica y el involucrado directo en cada caso (Concesionaria en temas de infraestructura y operaciones de los aeropuertos internacionales, Aerolíneas en caso de apertura de nuevas rutas y similares).

Se conoce la existencia de convenios que puedan abrir oportunidades para asesorías de expertos nacionales y/o internacionales, más se desconoce si se están implementando de manera conjunta con entidades que se vean afectadas por estos mismos convenios.

Aún cuando el CAN no sea el órgano en que convergen distintas entidades no directamente relacionadas con la Aeronáutica Civil pero afectadas por las decisiones de este rubro, es necesario considerar la opción de incluirlas en la toma de decisiones de la Aeronáutica Civil del país ya se a través del uso de Ministerios, Comités u otras organizaciones ya existentes que reunan a gran parte de los afectados por dichas decisiones.

La investigación evidencia que el involucramiento de entidades diversas como Turismo, Relaciones exteriores, Concesionarias y otros han logrado el cumplimiento de logros realmente importantes en materia de desarrollo de la Aeronáutica Civil de otros países y que ameritaría ser replicadas en Honduras, considerando que hay disponibilidad de información pública sobre la implementación en otros países y sus casos de éxito y aprovechando los convenios de cooperación internacional existentes entre países. 


\section{PROPUESTAS}

Realizar una investigación profunda, objetiva y precisa sobre otras opciones de inclusión de entidades no directamente relacionadas con la Aeronáutica Civil, pero que se ven afectadas por las decisiones en torno a ella, distintas a Órganos similares al CAN. Algunos temas que se sugiere se deberían considerar para nuevas investigaciones sobre la inclusión de otras entidades en la toma de decisiones en Aeronáutica Civil en Honduras incluyen: Estrategias entre dependencias del estado y dependencias privadas involucradas con el rubro aeronáutico, Aspectos en los que debería ser fortalecida la Autoridad Aeronáutica para hacer frente a un escenario con una mayor apertura para entidades relacionadas.

Hacer un estudio sobre herramientas tecnológicas disponibles a nivel nacional o internacional que permitan que la Autoridad Aeronáutica pueda obtener información clave de la entidades que podrían verse afectadas por determinada decisión sin necesidad de invertir tiempo y recursos en formar un órgano específico para ello.

\section{BIBLIOGRAFÍA}

- Ulloa Cadalso, A., \& Flores Moncada, A. (2016). El Consejo Aeronáutico Nacional: Análisis de su situación actual en el marco institucional de la Aeronáutica Civil Hondureña. Ciencias Espaciales, 7(2), 74-95. doi:http:// dx.doi.org/10.5377/ce.v7i2.2521

- Ley de Aeronáutica Civil de Honduras, Congreso Nacional de Honduras 19.05.2004.

- Ley de Aeronáutica Civil de Republica Dominicana, Congreso Nacional de República Dominicana 22.11.1969.

- Ley de Aviación Civil de la Republica Dominicana, Congreso Nacional de República Dominicana 28.12.2006.

- Hernández Sampieri, R., Fernández Collado, C., \& Baptista Lucio, P. (op. 2010). Metodología de la investigación (5a. ed.). México, Madrid [etc.]: McGraw-Hill.

- Fusiona y reorganiza diversos servicios relacionados con la aviación civil. Leyes de la Contraloría General de la República de Chile (Ministerio de Hacienda de Chile 06.04.1960). 
- Espacio Ecuador. Consejo Nacional de Aviación Civil de Ecuador. 2014. Página web. http://www.cnac.gob.ecl

- Junta de Aeronáutica Civil de Chile. 2014. Página web.http://www.jac-chile.cl

- Oficina Presidencial de Tecnologías de la Información y Comunicación (OPTIC). Junta de Aviación Civil de República Dominicana. 2014. Página web.http:// www.jac.gob.do

- Dirección General de Aeronáutica Civil de Honduras. Asesoría Legal. 2014. Página web.http://www.dgachn.org/asesorialegal.html 